\title{
Selective vagal influences at the gastroduodenal junction in sheep
}

\author{
$\mathrm{CH}$ Malbert, Y Ruckebusch
}

\author{
with the technical assistance of G Costes, JP Serthelon \\ LA INRA-Physiologie, Ecole Nationale Vétérinaire, \\ 23, chemin des Capelles, 31076 Toulouse Cedex, France
}

Introduction - The aim of this study was to define the role of the vagus nerves on the phasic and tonic motor activity of the gastroduodenal junction. The role of the vagus was examined by studying: 1) the effects of thoracic vagotomy in sheep maintained on a liquid diet, and 2) the changes obtained during transient cooling of the cervical vagus nerve in conscious sheep.

Materials and Methods - The motility of the antroduodenal area was assessed from a side hole catheter assembly using a low-compliance pneumohydraulic perfusion system. The left cervical vagus was severed in 2 sheep and a cooling jacket (Gleysteen et $a l, 1983)$ was placed around the right cervical vagus. The thoracic vagus nerves were sectioned in 2 other sheep already maintained on a liquid diet (Ruckebusch and Malbert, 1987).

Results et Discussion - Before vagotomy and during the phase of duodenal irregular spiking activity (ISA), the pylorus smooth muscle tone was $31.2 \pm 5.0 \mathrm{~mm} \mathrm{Hg}(n>100)$. The pyloric tone gradually increased by $20 \%$ during the phase of regular spiking activity (RSA) and exhibited phasic motor activity at intervals of $6.9 \pm$
$0.3 \mathrm{~s}$. The pyloric tone was minimal $(26.1 \pm 3.2$ $\mathrm{mm} \mathrm{Hg}$ ) during the subsequent phase of quiescence. These values remained unchanged after sectioning the left vagus. Hyperactivity of the abomasum associated with an RSA phase on the proximal duodenum was triggered by right vagal cooling. When the vagus was blocked, the pyloric tone was depressed and its phasic motor activity suppressed. The frequency of antral contractions was increased by $75 \%$ immediately after thoracic vagotomy and remained higher than control values over the whole recording period ( $>3 \mathrm{wk}$ ). The interval between successive RSA phases $(85 \pm 17$ versus $90 \pm$ $14 \mathrm{~min}$ ) was not, however, significantly modified.

It is concluded that the vagus nerves decrease the frequency of antroduodenal contractions by means of inhibitory in-fluences. The effects are completely dit-ferent from those observed on the pylorus.

Gleysteen JJ, Esser MJ, Myrvik AL (1983) Gastroenterology 85, 578-583

Ruckebusch Y, Malbert CH (1987) Dig Dis Sci32, 26A

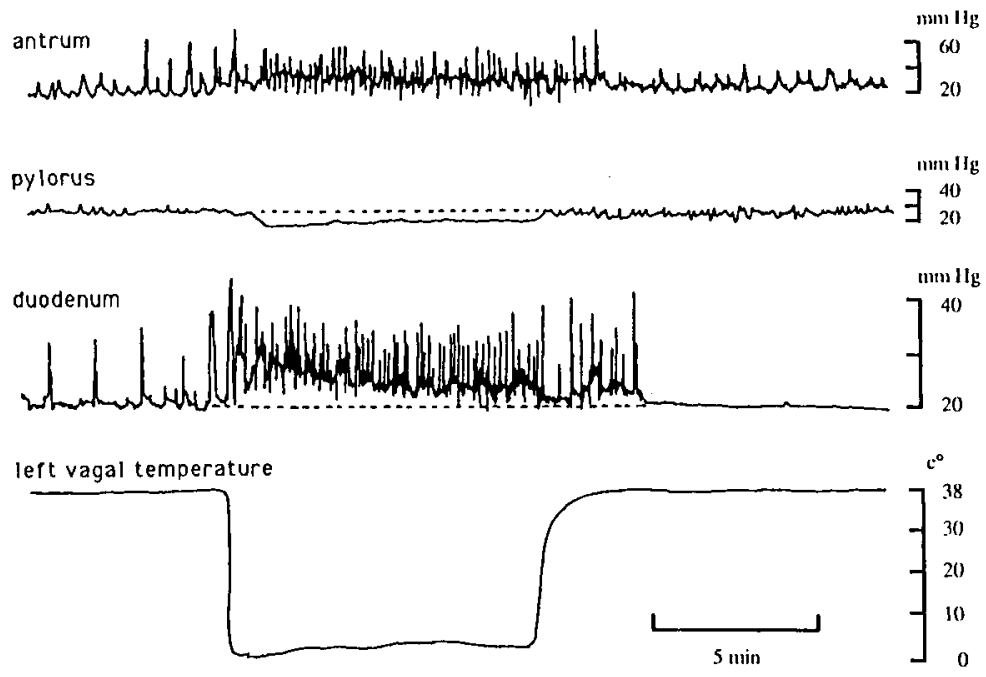

Fig 1. Pressure changes in the gastroduodenal area during left vagal cooling in sheep. Antral and duodenal pressures were increased whereas the intrapyloric pressure was lowered, indicating relaxation of the pylorus. 